\title{
lon chromatography using anion exchangers modified with heparin
}

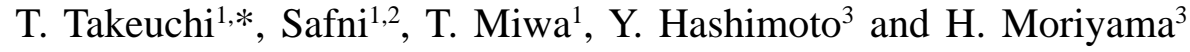 \\ ${ }^{1}$ Department of Chemistry, Faculty of Engineering, Gifu University, 1-1 Yanagido, Gifu 501-1193, Japan \\ ${ }^{2}$ On leave from Department of Chemistry, Faculty of Mathematics and Natural Sciences, \\ Andalas University, Padang, West Sumatra, Indonesia \\ ${ }^{3}$ Separation Center, Tosoh, 4560 Kaisei-cho, Shinnanyo-shi 746-8501, Japan
}

\begin{abstract}
Retention behavior of inorganic anions and cations on anion exchangers modified with heparin has been investigated. Retention of anions on the anion exchanger was remarkably reduced after the modification with heparin. The cations of the eluent affected the retention behavior of ions. The retention factor of anions decreased with decreasing eluent concentration when sodium sulfate and magnesium sulfate were used as the eluent, whereas it increased with decreasing eluent concentration when aluminium sulfate, copper sulfate and sulfuric acid were used as the eluent. When copper sulfate was used as the eluent, both anions and cations were retained on the modified stationary phase and simultaneous separation of both anions and cations were carried out. Cations as well as non-UV-absorbing anions were indirectly visualized by copper ions.
\end{abstract}

Key words. Ion chromatography - heparin - simultaneous separation of anions and cations.

\section{Introduction}

Anions and cations have usually been analyzed separately by using different separation columns in ion chromatography. If anions and cations can be simultaneously separated in a single chromatographic run, agents and solvents can be saved and analysis time may be shortened. Simultaneous separation of both anions and cations in a single chromatographic run has been challenged in various ways in ion chromatography [1-11]. Mixed bed of anion-exchange and cation-exchange materials $[1,2]$ or the use of anionexchange and cation-exchange columns in tandem [3-6] can retain both anions and cations. Amphoteric stationary phases such as alumina [7] as well as zwitterionic stationary phases such as bile acids [8] also allow simultaneous separation of anions and cations on a single stationary phase. A weak anion exchanger allows the simultaneous separation of anions and cations using tartaric acid as the eluent, where anions are separated in the ion-exclusion mode, whereas cations are separated in the cation-exchange mode [9]. The formation of anionic metal chelates opened up the possibility for simultaneous determination of these metal chelates and common inorganic anions on an anion-exchange column. A separation of anions and metal-EDTA (ethylenediaminetetraacetic acid) complexes is demonstrated [10,11].

It was found by the authors that anion-exchangers modified with anionic polysaccharides such as chondroitin sulfate or heparin showed unusual retention behavior for anions $[12,13]$, e.g., the retention factor of analyte anions decreased with decreasing eluent concentration. It is presumed that such unusual retention behavior is attributed to the presence of both cationic and anionic sites on the modified surfaces. In other words, it is expected that anion exchangers modified with anionic polysaccharides retain both anions and cations.
This paper will examine the retention behavior of both anions and cations on anion exchangers modified with heparin.

\section{Experimental}

\section{Apparatus}

A microcolumn liquid chromatograph was comprised from an MF-2 Microfeeder (Azumadenki Kogyo, Tokyo, Japan) equipped with an MS-GAN 050 gas-tight syringe $(0.5 \mathrm{~mL}$; Ito, Fuji, Japan), an ML-522 microvalve injector with an injection volume of 0.2 or $0.11 \mu \mathrm{L}$ (Jasco, Tokyo, Japan), a $100 \times 0.32 \mathrm{~mm}$ i.d. microcolumn, and a UVIDEC-100V UV detector (Jasco, Tokyo, Japan). The UV detector was operated at 210 or $200 \mathrm{~nm}$, and the data were acquired by a Chromatopac C-R4AX (Shimadzu, Kyoto, Japan) or a Computer Aided Chromatography data processor (Nippon Filcon, Tokyo, Japan). Size-exclusion chromatography of heparin was carried out by using $\mathrm{TSK}_{\mathrm{gel}} \mathrm{GMPW}_{\mathrm{XL}}$ columns $(300 \times 7.8 \mathrm{~mm}$ i.d. $\times 2$; Tosoh, Tokyo, Japan $)$ and $0.2 \mathrm{M}$ sodium nitrate as the eluent.

The microcolumns were prepared in the laboratory from fused-silica tubing as reported previously [14]. The packing material examined in this work was $\mathrm{TSK}_{\mathrm{gel}}$ IC-Anion SW (Tosoh), a silica-based anion exchanger with ca. $0.4 \times 10^{-3}$ equivalent $\mathrm{g}^{-1}$.

\section{Reagents}

The reagents employed were of guaranteed-reagent-grade and were obtained from Nacalai Tesque (Kyoto, Japan), unless otherwise noted. Sodium salt of heparin was also obtained from Nacalai Tesque. The reagents were used 


\section{Original articles}

without any further treatment. Purified water was prepared in the laboratory by using a Mill-Q Plus system (Millipore, Molsheim, France). The eluents were prepared using the purified water.

\section{Modification with heparin}

The IC Anion-SW column was first washed with water at a flow rate of $4.2 \mu \mathrm{L} \mathrm{min}{ }^{-1}$ for $10 \mathrm{~min}$ and with $10 \mathrm{mM}$ sodium sulfate at the same flow rate for $2 \mathrm{~h}$. Aqueous solution of $1.0 \%$ heparin was then passed through the column at a flow rate of $4.2 \mu \mathrm{L} \mathrm{min}{ }^{-1}$ for $2 \mathrm{~h}$, followed by washing with water at the same flow rate for ca. $30 \mathrm{~min}$.

\section{Results and discussion}

\section{Modification with heparin}

The expected structure of heparin is illustrated in figure 1 . Since heparin has various anionic groups in its unit as in figure 1, it can be introduced into anion exchangers by electrostatic interaction. Since all of the anionic groups do not interact with the anion-exchange sites of the packing, a portion of anionic groups of heparin are remained free and they affect the retention of analyte ions. The repulsion of analyte anions from the modified surface may depend on the $\mathrm{pH}$ and the ionic strength of the eluent.

The mass distribution of heparin employed in this work is illustrated in figure 2. From the size-exclusion chromatography data, the weight-average molecular weight of heparin employed was calculated to be $2.2 \times 10^{4}$ by using poly(ethyleneoxide) (PEO) as the standard, whereas the

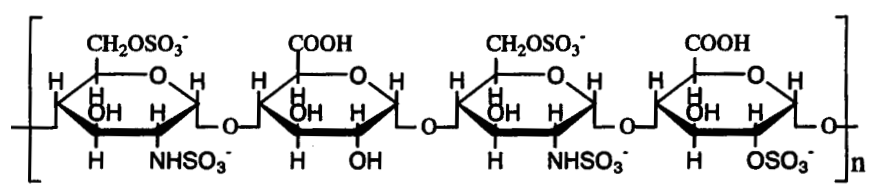

\section{heparin}

Fig. 1. Expected structure of heparin.

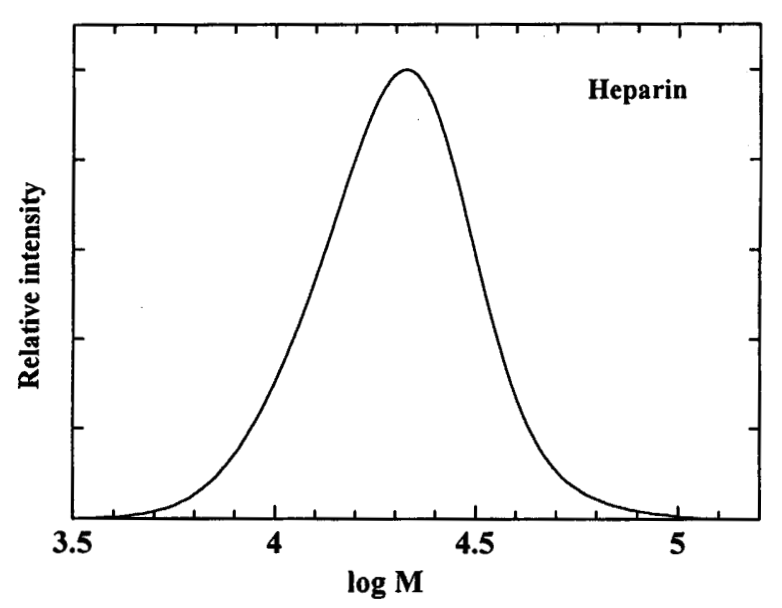

Fig. 2. Mass distribution of heparin. Calibrated with PEO. molecular weight of PEO completely excluded from the pore of the IC-Anion-SW packing is $(5-6) \times 10^{4}$. From these estimations, it is presumed that heparin can enter the pore of the packing to some depth depending on the size and a portion of the inner part of the packing is remained unmodified. In other words, the modified packing possesses both negative charges due to free anionic groups of heparin and positive charges due to the original bare anion-exchange sites. It is therefore expected that both anions and cations are retained on the modified stationary phase under some appropriate conditions.

\section{Effect of modification on retention of anions}

Figure 3 demonstrates the separation of iodate, nitrate, iodide and thiocyanate on unmodified (A) and modified stationary phases (B) using $10 \mathrm{mM}$ sodium sulfate as the eluent. It is seen that the retention of anions are remarkably reduced after the modification with heparin. This is because the number of anion-exchange sites is reduced by the modification and the analyte anion undergo electrostatic repulsion from the modified surface.

The cation of the eluent strongly influenced the retention of analyte anions on the modified stationary phase. Sulfates with various counter cations were employed as the eluent, and the effect of the counter cation of the sulfate on the retention was examined. Sodium sulfate, sulfuric acid, magnesium sulfate, copper sulfate and aluminum sulfate were examined in this work. Figure 4 plots the retention factor $(k)$ of nitrate as analyte anion versus the sulfate concentration in the eluent. In common ion chromatography the retention time increases with decreasing eluent concentration, as demonstrated for the unmodified stationary phase. As for the modified stationary phase, the retention behavior strongly depended on the counter cation of the sulfate eluents. $k$ increased with decreasing eluent concentration for copper sulfate, aluminum sulfate and sulfuric acid, whereas it decreased with decreasing eluent concentration for sodium sulfate and magnesium sulfate. The latter retention behavior

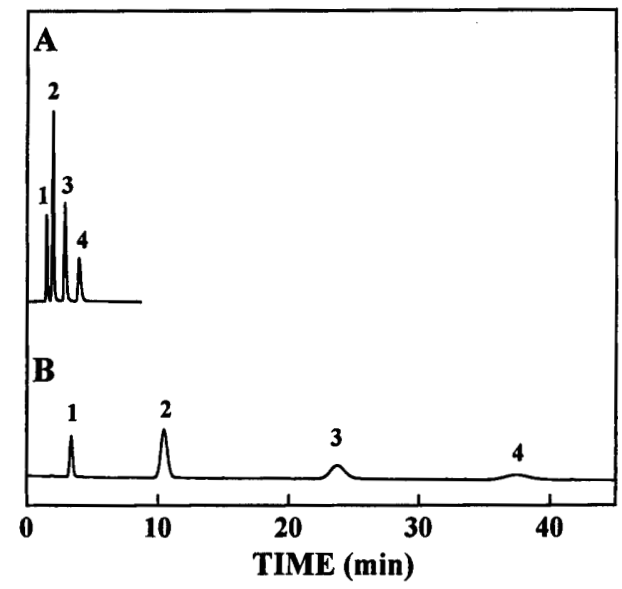

Fig. 3. Separation of UV-absorbing anions on the modified (A) and unmodified anion exchanger (B). Columns, TSK $_{\text {gel }}$ IC-Anion SW $(100 \times 0.32 \mathrm{~mm}$ i.d. $)$, modified with heparin (A), unmodified (B); eluent, $10 \mathrm{mM}$ sodium sulfate; flow rate, $4.2 \mu \mathrm{L} \mathrm{min}{ }^{-1}$; wavelength of UV detection: $210 \mathrm{~nm}$; analytes, $1=$ iodate, $2=$ nitrate, $3=$ iodide, $4=$ thiocyanate; injection volume, $0.2 \mu \mathrm{L}$. 


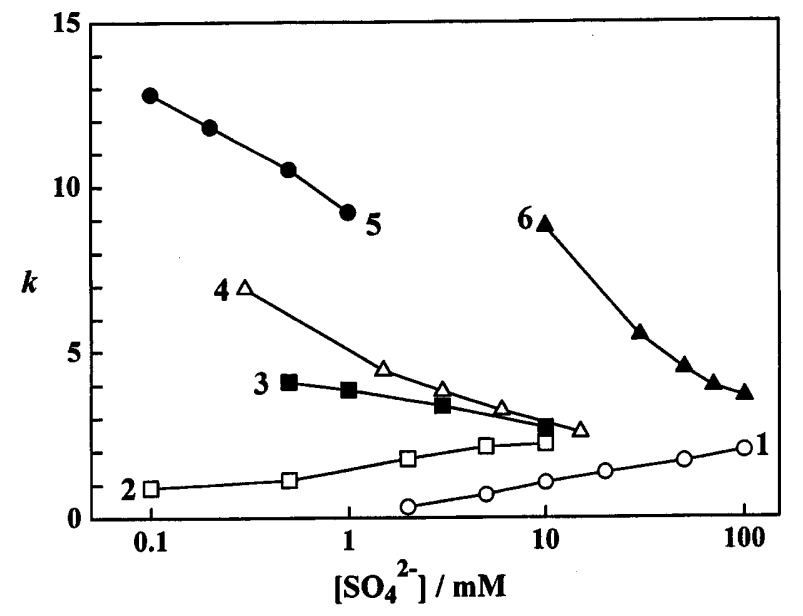

Fig. 4. Retention factor versus the sulfate concentration in the eluent. Columns, $100 \times 0.32 \mathrm{~mm}$ i.d., TSK $_{\text {gel }}$ IC-Anion SW modified with heparin (1-5) or unmodified (6); eluents, $1=$ sodium sulfate, $2=$ magnesium sulfate, $3=$ copper sulfate, $4=$ aluminum sulfate $5=$ sulfuric acid, $6=$ sodium sulfate; flow rate, $4.2 \mu \mathrm{L} \mathrm{min}^{-1}$; analyte, sodium nitrate; injection volume, $0.2 \mu \mathrm{L}$.

is unusual in ion chromatography. The smallest retention factor was observed for sodium sulfate eluent. It should be noted that when water was used as the eluent, analyte anions in figure 3 were not retained on the modified stationary phase which was beforehand conditioned with sodium sulfate.

\section{Simultaneous separation of anions and cations}

Since the anion exchanger modified with heparin possesses both anion- and cation-exchange sites, it is expected that both anions and cations are simultaneously retained on the modified stationary phase. Copper sulfate was selected as the eluent to visualize cations by indirect photometric detection. In case UV-absorbing anions were injected, both anions and cations could be monitored. Figure 5 demonstrates the chromatogram when a mixture of $2 \mathrm{mM}$ sodium, $2 \mathrm{mM}$ magnesium. $1 \mathrm{mM}$ chloride, $1 \mathrm{mM}$ bromide and $2 \mathrm{mM}$ sulfate is injected. The eluent employed is $1 \mathrm{mM}$ copper sulfate and the detection wavelength is $200 \mathrm{~nm}$. It is seen that both cations and anions are simultaneously separated. Sodium and magnesium ions appeared as the negative peaks owing to the depression of copper ion, whereas chloride and bromide appeared as positive peaks. A system peak due to copper sulfate is also seen in the chromatogram. Bromide appears as the positive peak because it absorbs $200 \mathrm{~nm}$. On the other hand, since the absorption of chloride at $200 \mathrm{~nm}$ is very weak, it is reasonable to conclude that chloride is indirectly visualized by copper ion. In common indirect photometric detection in ion chromatography, ions with the same charge as the UV-absorbing eluent ion are visualized, whereas those with the opposite charge cannot be seen [3]. Unfortunately, the detection mechanism is not certain, yet.

Under the conditions as in figure 5, other transparent ions such as cyanate, chlorate, chlorite and phosphate were also visualized as positive peaks. It is reported that both cations and anions could simultaneously be visualized by salicylate eluent when an amphoteric stationary phase such as alumina

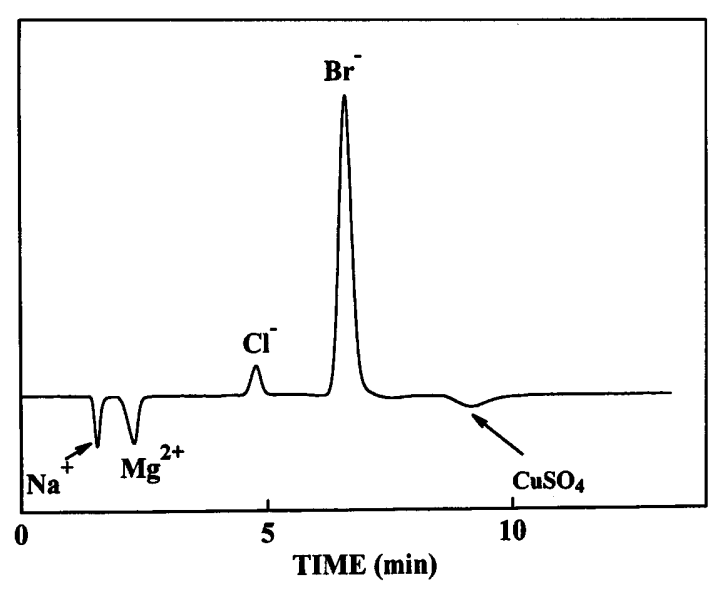

Fig. 5. Simultaneous separation of anions and cations. Column, TSK $_{\text {gel }}$ IC-Anion SW $(100 \times 0.32 \mathrm{~mm}$ i.d. $)$, modified with heparin; eluent, $1 \mathrm{mM}$ copper sulfate; flow rate, $4.2 \mu \mathrm{L} \mathrm{min}^{-1}$; analyte ions, $2 \mathrm{mM}$ sodium, $2 \mathrm{mM}$ magnesium, $1 \mathrm{mM}$ chloride, $1 \mathrm{mM}$ bromide and $2 \mathrm{mM}$ sulfate; injection volume, $0.2 \mu \mathrm{L}$; wavelength of UV detection, $200 \mathrm{~nm}$.

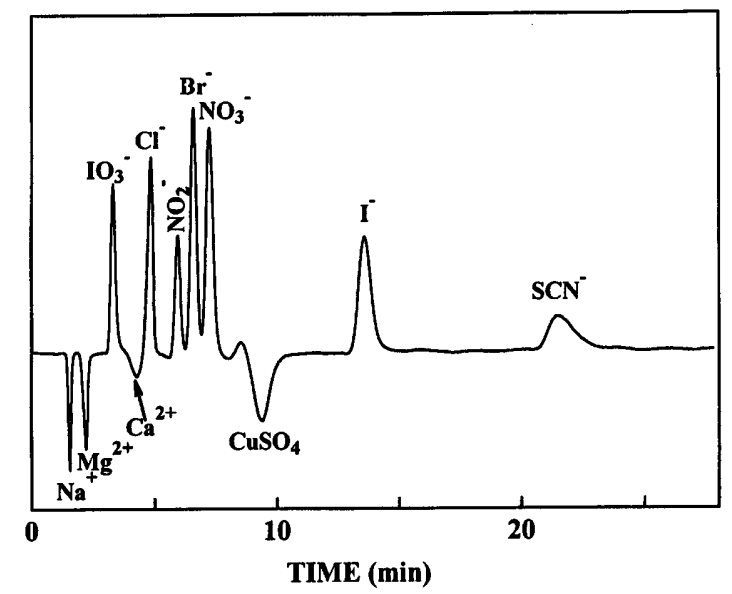

Fig. 6. Simultaneous separation of more components of anions and cations. Operating conditions as in figure 5 except for the analyte ions; analyte ions, $2.4 \mathrm{mM}$ sodium, $2 \mathrm{mM}$ magnesium, $2 \mathrm{mM}$ calcium, $0.2 \mathrm{mM}$ iodate, $2 \mathrm{mM}$ chloride, $0.2 \mathrm{mM}$ nitrite, $0.2 \mathrm{mM}$ bromide, $0.2 \mathrm{mM}$ nitrate, $0.2 \mathrm{mM}$ iodide, $0.2 \mathrm{mM}$ thiocyanate and $2 \mathrm{mM}$ sulfate.

was used, where cations appeared as positive peaks and anions appeared as negative peaks [7]. It is presumed that when both cation- and anion-exchange sites exist simultaneously, both anions and cations can be visualized by indirect photometric detection. In order to elucidate the detection mechanism using amphoteric stationary phases, more experiments will be required.

Under the conditions in figure 5 the repeatability of the retention time and the peak heights were measured. The relative standard deviation of the retention time and peak height for five successive measurements were $0.3-0.5 \%$ and $0.4-2.1 \%$, respectively. 


\title{
Original articles
}

Under the conditions in figure 5, the selectivity of monovalent cations were poor. Monovalent cations such as alkali metal ions and ammonium ion eluted too close to be separated. As for alkaline-earth metal ions such as magnesium, strontium and calcium eluted in this order. The selectivity of cations will be improved by careful selection of the molecular weight of modifier and/or the pore size of the packing.

More components of anions and cations are separated on the stationary phase modified with heparin in figure 6 . The elution order of the anions were the same as that of the unmodified stationary phase.

\section{Conclusion}

The retention behavior of inorganic anions on the anionexchanger modified with heparin was different from that observed under conventional ion chromatographic conditions. The retention of anions was strongly affected by the counter cation of the eluent. When copper sulfate was used as the eluent, both anions and cations were simultaneously separated. The present stationary phase may allow the separation of anions and/or cations by altering the eluent. The elucidation of the retention mechanism is being investigated.

\section{References}

1. Pietrzyk, D. J.; Brown, D. M. Anal. Chem. 1986, 58, 25542557.

2. Brown, D. M.; Pietrzyk, D. J. J. Chromatogr. 1989, 466, 291300.

3. Small, H.; Miller, T. E. Jr. Anal. Chem. 1982, 54, 462-469.

4. Iskandarani, Z.; Miller, T. E. Jr. Anal. Chem. 1985, 57, 15911594.

5. Tarter, J. G. J. Chromatogr. 1986, 367, 191-194.

6. Gan, D. -C.; Tarter, J. G. J. Chromatogr. 1987, 404, 285-291.

7. Takeuchi, T.; Suzuki, E.; Ishii, D. Chromatographia 1988, 25, 480-482.

8. Hu, W.; Haraguchi, H. Anal. Chem. 1994, 66, 765-767.

9. Tanaka, K.; Ohta, K.; Matsushita, S.; Miyanaga, A. J. Chromatogr. A 1994, 671, 239-248.

10. Matsushita, S. J. Chromatogr. 1984, 312, 327-336.

11. Hayakawa, K.; Sawada, T.; Shimbo, K.; Miyazaki, M. Anal. Chem. 1987, 59, 2241-2245.

12. Takeuchi, T.; Safni; Miwa, T. J. Chromatogr. A 1997, 789, 201-206.

13. Takeuchi, T.; Safni; Miwa T.; Hashimoto, Y.; Moriyama, H. J. Chromatogr. A 1998, in press.

14. Takeuchi, T.; Ishii, D; J. Chromatogr. 1981, 213, 25-32.

\section{Determination of nonionic aliphatic and aromatic polyethoxylated surfactants in environmental aqueous samples}

\author{
A. Marcomini ${ }^{1 *}$, G. Pojana ${ }^{1}$, L. Patrolecco ${ }^{2}$ and S. Capri ${ }^{2}$ \\ ${ }^{1}$ Department of Environmental Sciences, University of Venice, Calle Larga S. Marta 2137, 30123 Venice, Italy \\ ${ }^{2}$ Water Research Institute (IRSA-CNR), Via Reno 1, 00198 Rome, Italy
}

\begin{abstract}
Nonionic surfactants of the polyethoxylate type are environmentally relevant because of their ubiquitous presence in raw and treated, municipal and industrial, wastewaters. Until today the standard methods currently used for the routine determination of nonionic surfactants in environmental matrices are cumulative methods (BIAS, CTAS, PPAS, TAS), which are poorly reliable, particularly in terms of accuracy, and do not supply any structural information on the ethoxymeric and homolog composition, as well as on the chain length of the hydrophobic moiety. This paper describes a specific analytical procedure for the simultaneous specific determination of polyethoxylate aliphatic (AE) and aromatic (APE) nonionics in aqueous matrices. The analytes were isolated from water samples by solid phase extraction with graphitized carbon black and then derivatized with 1-naphthylisocyanate (NIC). AE and APE were separated by reversed-phase HPLC combined with fluorescence detection (RP-HLPC/FL). The analytical procedure was applied to the monitoring of these two classes of surfactants in a municipal sewage treatment plant. The method allows rapid, precise and reliable determination of AE and APE in environmental samples at concentrations as low as $0.1 \mu \mathrm{g} / \mathrm{L}$.
\end{abstract}

Key words. Aliphatic alcohol polyethoxylates - nonylphenol polyethoxylates - nonionic surfactants - reversed phase HPLC environmental aqueous samples.

* Correspondence and reprints

Received November 03, 1997; revised January 13, 1998; accepted January 14, 1998. 